Document downloaded from:

http://hdl.handle.net/10251/65895

This paper must be cited as:

Islam, SMR.; Ullah, S.; Lloret, J.; Ullah, N.; Kwak, KS. (2015). SIR performance evaluation of MB-OFDM UWB system with residual timing offset. Electronics Letters. 51(5):427-429. doi:10.1049/el.2014.3967.

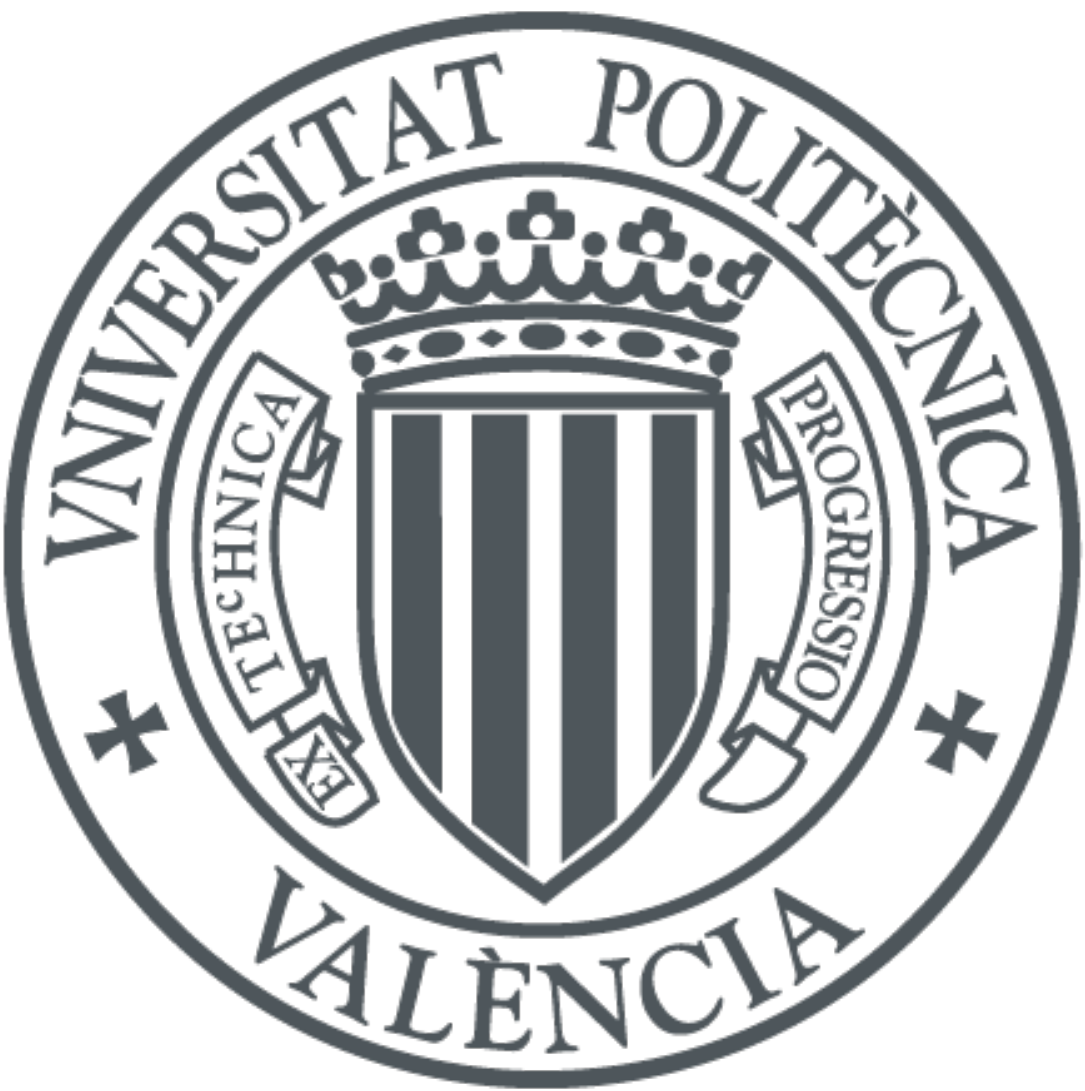

The final publication is available at

http://dx.doi.org/10.1049/el.2014.3967

Copyright Institution of Engineering and Technology (IET)

Additional Information 


\section{SIR performance evaluation of MB-OFDM UWB system with residual timing offset}

S.M.R. Islam $^{\bowtie}$, S. Ullah, J. Lloret, N. Ullah and K.S. Kwak

Signal-to-interference ratio (SIR) performance of a multiband orthogonal frequency division multiplexing ultra-wideband system with residual timing offset is investigated. To do so, an exact mathematical derivation of the SIR of this system is derived. It becomes obvious that unlike a cyclic prefixing based system, a zero padding based system is sensitive to residual timing offset.

Introduction: Multiband orthogonal frequency-division multiplexing (MB-OFDM) ultra-wideband (UWB) uses zero padding (ZP) instead of cyclic prefixing $(\mathrm{CP})$, since the use of $\mathrm{ZP}$ confirms symbol recovery irrespective of the channel zero locations vis-à-vis superior computational complexity. There are several preamble based symbol synchronisation methods for ZP MB-OFDM systems [1-10]. The best practice in achieving satisfactory symbol synchronisation in ZP MB-OFDM UWB system is to follow a three-step approach consisting of sync detection, coarse-timing estimation and fine-timing estimation. Nevertheless, the use of further timing error or residual timing offset hereafter is obvious. The effect of residual fractional timing errors in terms of signal-to-interference ratio (SIR) is characterised in [11] for CP-based OFDM system. Sensitivity of a ZP MB-OFDM UWB system to synchronisation error has been analysed in [12], where the system is found sensitive to carrier frequency offset. However, the effect of integer timing errors on SIR performance of the available timing estimation algorithms has always been ignored. Given this perspective, we explore an exact mathematical analysis to show the impact of the residual timing offset on SIR in ZP MB-OFDM UWB system.

System model: An MB-OFDM system transmits information bits across multiple sub-bands to utilise frequency diversity. A specific time-frequency code (TFC) is used for this purpose. Moreover, different sets of training sequences are defined for different band groups. There is a packet layer convergence protocol preamble of 18 or 30 OFDM symbols inside each band group. Each OFDM symbol in a band consists of $N$ subcarriers, $L$ number of guard samples with $L=L_{\text {pre }}+L_{\text {suf, }}$, where $L_{\text {pre }}$ and $L_{\text {suf }}$ are zeros appended as prefix and suffix of the OFDM symbol, respectively.

Under the above system assumption and having perfect frequency synchronisation, we can have the expression of time-domain received samples corresponding to $i$ th sub-band $y_{i}(n)$ as

$$
\begin{aligned}
y_{i}(n)= & s_{i}(n) * h_{i}(n)+w_{i}(n) \\
& 0 \leq n \leq N+L-1 \text { and } 1 \leq i \leq 3
\end{aligned}
$$

where the symbols $s_{i}(n)$ and $h_{i}(n)$ are the transmitted signal samples and sample-spaced impulse response of the UWB channel, respectively. Note that we have assumed that TFC is performed across just three OFDM symbols. The noise $w_{i}(n) \in C N\left(0, \sigma_{w}^{2}\right)$. Here, $C N\left(0, \sigma_{w}^{2}\right)$ represents circularly symmetric complex Gaussian process with mean 0 and variance $\sigma_{w}^{2}$. We perform the initial 'synch' detection and coarse timing based on the algorithms outlined in [1].

Impacts of residual timing offset: We consider that there is a timing error of $d$ in the received vector of time-domain samples after the coarse timing step. Now, we perform overlap and added (OLA) operation by adding the last $L_{\text {suf }}$ number of samples with the first $L_{\text {suf }}$ number of samples. After OLA operation, the resulting $N \times 1$ vector $\boldsymbol{y}_{i}$ is represented as

$$
\boldsymbol{y}_{i}=\boldsymbol{S} \boldsymbol{J}^{\mathrm{d}} \boldsymbol{T h}_{i}+\boldsymbol{w}
$$

where $\boldsymbol{h}_{i}=\left[h_{i, 0}, h_{i, 1}, \ldots, h_{i, M-1}\right]^{\mathrm{T}}$ is the discrete time $M$-tap channel impulse response of the $i$ th UWB band; $\boldsymbol{w} \in C N\left(0, \sigma_{w}^{2} \boldsymbol{I}_{N}\right) ; \boldsymbol{J}$ is an $N \times N$ circular shift matrix given by

$$
\boldsymbol{J}=\left[\begin{array}{cc}
\mathbf{0}_{1 \times(N-1)} & 1 \\
\boldsymbol{I}_{(N-1)} & \mathbf{0}_{(N-1) \times 1}
\end{array}\right]
$$

$\boldsymbol{T}$ is an $N \times M$ tail zero insertion matrix, defined as

$$
\boldsymbol{T}=\left[\begin{array}{c}
\boldsymbol{I}_{M} \\
\mathbf{0}_{(N-M) \times M}
\end{array}\right]
$$

$\boldsymbol{S}$ is an $N \times N$ circulant matrix of training samples. The maximum likelihood estimate of $d$ becomes

$$
\hat{d}=\max _{d} \sum_{i=1}^{3}\left\|\boldsymbol{T}^{\mathrm{H}} \boldsymbol{J}^{-d} \hat{\boldsymbol{h}}_{\boldsymbol{i}}\right\|^{2}=\max _{d} \sum_{i=1}^{3} \sum_{m=d}^{d+M-1}\left|\hat{h}_{i, m}\right|^{2}
$$

Now, we consider that there is a residual timing error of $d_{\mathrm{r}}$ in the received vector of time-domain samples after the fine timing step. For notational ease, we omit the subscript ' $i$ ' in the subsequent mathematical expressions.

With a similar approach followed in obtaining (2), we do the OLA operation and can then have the $n$th time-domain sample of the received vector as

$$
r_{n}=\sum_{m=0}^{M-1} h_{m} s\left((n-m)_{N}\right)+w_{n}=z_{n}+w_{n}, \quad 0 \leq n \leq N-1
$$

Owing to the residual error of $d_{\mathrm{r}}$, the $d_{\mathrm{r}}$ samples of the current OFDM symbol will be missed and this will in turn affect the next received symbol. Suppose, the missing samples are $r_{0}, \ldots, r_{d_{\mathrm{r}}}$. As a result, the $n$th sample of the received signal is given by

$$
v_{n}=z_{\left(n+d_{\mathrm{r}}\right)_{N}} \gamma_{n}+x_{n}
$$

Here, we did not consider the noise to reveal the effect of interference only. Symbols $\gamma_{n}$ and $x_{n}$ are as follows:

$$
\gamma_{n}= \begin{cases}1, & 0 \leq n \leq N-d_{\mathrm{r}}-1 \\ 0, & N-d_{\mathrm{r}} \leq n \leq N-1\end{cases}
$$

and

$$
x_{n}=\left\{\begin{array}{cc}
0, & 0 \leq n \leq N-d_{\mathrm{r}}-1 \\
z^{0}(n), & N-d_{\mathrm{r}} \leq n \leq N-1
\end{array}\right.
$$

In (7), $z^{0}(n)$ denotes the $n$th time-domain received sample in the zero prefix region of the current OFDM symbol.

By taking all the samples, we constitute the corresponding column vectors $\boldsymbol{v}, \boldsymbol{h}, \boldsymbol{s}, \mathbf{x}, \boldsymbol{\gamma}$ and $\boldsymbol{z}$, each of size $N$, and whose fast Fourier transforms (FFTs) are denoted by $\boldsymbol{V}, \boldsymbol{H}, \boldsymbol{X}, \boldsymbol{S}, \boldsymbol{\Gamma}$ and $\boldsymbol{Z}$, respectively. Thus, we can present the $k$ th element of $\boldsymbol{\Gamma}$ as

$$
\begin{aligned}
\Gamma_{k} & =\sum_{n=0}^{N-1} \gamma_{n} \mathrm{e}^{-\mathrm{j} 2 \pi n k / N}=\sum_{n=0}^{L-d_{\mathrm{r}}-1} \gamma_{n} \mathrm{e}^{-\mathrm{j} 2 \pi n k / N} \\
& =\left\{\begin{array}{cc}
\frac{1-\mathrm{e}^{\mathrm{j} 2 \pi d_{r} k / N}}{1-\mathrm{e}^{-\mathrm{j} 2 \pi k / N}}, & 1 \leq k \leq N-1 \\
L-d_{\mathrm{r}}, & k=0
\end{array}\right.
\end{aligned}
$$

And we also have the $k$ th element of $\boldsymbol{Z}$ as

$$
Z_{k}=H_{k} S_{k} \mathrm{e}^{\mathrm{j} 2 \pi d_{\mathrm{r}} k / N}
$$

Collecting (5), (8) and (9), the $k$ th element of $\boldsymbol{V}$ is given by

$$
\begin{aligned}
V_{k}= & \left(Z_{k} * \Gamma_{k}\right)+X_{k}=\frac{1}{N} \Gamma_{0} H_{k} S_{k} \mathrm{e}^{\mathrm{j} 2 \pi d_{\mathrm{r}} k / N} \\
& +\frac{1}{N} \sum_{n=1}^{N-1} \Gamma_{n} H_{(k-n)_{L}} S_{(k-n)_{N}} \mathrm{e}^{\mathrm{j} 2 \pi d_{\mathrm{r}}(k-n) / N}+X_{k}
\end{aligned}
$$

In the above equation, only the first term corresponds to signal component; the second and third terms correspond to intercarrier interference. Let us denote the second term in (10) as

$$
I_{k}=\frac{1}{N} \sum_{n=1}^{N-1} \Gamma_{n} H_{(k-n)_{L}} S_{(k-n)_{N}} \mathrm{e}^{\mathrm{j} 2 \pi d_{\mathrm{r}}(k-n) / N}
$$

where the $H_{i}$ and $S_{i}$ 's are assumed to be jointly and individually uncorrelated.

From (10), we can have the total interference power as

$$
E\left[\left|I_{k}+X_{k}\right|^{2}\right]=E\left[\left|I_{k}\right|^{2}\right]+E\left[\left|X_{k}\right|^{2}\right]+2 \operatorname{Re}\left\{E\left[\left|I_{k} X_{k}^{*}\right|\right]\right\}
$$

Using Perseval's theorem and after trivial algebraic manipulations, the power of $I_{k}$ in (12) can be shown as

$$
E\left[\left|I_{k}\right|^{2}\right]=\frac{d_{\mathrm{r}}\left(N-d_{\mathrm{r}}\right) \sigma_{\mathrm{h}}^{2} \sigma_{\mathrm{s}}^{2}}{N^{2}}
$$


where symbols $\sigma_{\mathrm{h}}^{2}$ and $\sigma_{\mathrm{s}}^{2}$ represents the variances of channel coefficients and signals. The power of $X_{k}$ in (12) can be calculated as

$$
E\left[\left|X_{k}\right|^{2}\right]=E\left[\left|x_{n}\right|^{2}\right]=\sum_{r=0}^{d_{r}-1} \sum_{m=r+1}^{M-1} E\left\{\left|h_{m}\right|^{2}\left|s_{N-m+r}\right|^{2}\right\}
$$

where

$$
E\left\{\left|s_{N-m+r}\right|^{2}\right\}=\frac{\sigma_{\mathrm{s}}^{2}}{N}
$$

Thus, (14) can be written as

$$
E\left[\left|X_{k}\right|^{2}\right]=\frac{\sigma_{\mathrm{s}}^{2}}{N} \sum_{r=0}^{d_{0}-1} \sum_{m=r+1}^{M-1} E\left\{\left|h_{m}\right|^{2}\right\}=\frac{\sigma_{\mathrm{s}}^{2}}{N} \sum_{r=0}^{M-1} \sum_{m=r+1}^{M-1} \sigma_{h_{m}}^{2}
$$

Using (13), (15) and finding the cross-correlation terms in (12), we can write the total interference power as

$$
\begin{aligned}
E\left[\left|I_{k}+X_{k}\right|^{2}\right]= & \frac{d_{\mathrm{r}}\left(N-d_{\mathrm{r}}\right) \sigma_{\mathrm{h}}^{2} \sigma_{\mathrm{s}}^{2}}{N^{2}}+\frac{\sigma_{\mathrm{s}}^{2}}{N} \sum_{r=0}^{d_{\mathrm{r}}-1} \sum_{m=r+1}^{M-1} \sigma_{h_{m}}^{2} \\
& +\frac{2\left(N-d_{\mathrm{r}}\right) \sigma_{\mathrm{s}}^{2}}{N^{2}} \sum_{r=0}^{d_{\mathrm{r}}-1} \sum_{m=r+1}^{M-1} \sigma_{h_{m}}^{2} \\
= & \frac{\left(N-d_{\mathrm{r}}\right) \sigma_{\mathrm{h}}^{2} \sigma_{\mathrm{s}}^{2}-\left(\left(\left(n-2 d_{\mathrm{r}}\right) \sigma_{\mathrm{s}}^{2}\right) / \sigma_{h}^{2}\right) \sum_{r=0}^{d_{\mathrm{r}}-1} \sum_{m=r+1}^{M-1} \sigma_{h_{m}}^{2}}{N^{2}}
\end{aligned}
$$

On the other hand, the signal power can be calculated as

$$
\begin{aligned}
& E\left[\left(\frac{1}{N} \Gamma_{0} H_{k} S_{k} \mathrm{e}^{\mathrm{j} 2 \pi d_{\mathrm{r}} k / N}\right)\left(\frac{1}{N} \Gamma_{0} H_{k} S_{k} \mathrm{e}^{\mathrm{j} 2 \pi d_{\mathrm{r}} k / N}\right)^{*}\right] \\
& =\frac{1}{N^{2}}\left(N-d_{\mathrm{r}}\right)^{2} \sigma_{\mathrm{h}}^{2} \sigma_{\mathrm{s}}^{2}
\end{aligned}
$$

Thus, dividing (17) by (16), the expression of SIR becomes

$$
R=\frac{\left(N-d_{\mathrm{r}}\right)^{2}}{\left(N-d_{\mathrm{r}}\right) d_{\mathrm{r}}-\left(\left(\left(n-2 d_{\mathrm{r}}\right)\right) / \sigma_{\mathrm{h}}^{2}\right) \sum_{r=0}^{d_{\mathrm{r}}-1} \sum_{m=r+1}^{L-1} \sigma_{h_{m}}^{2}}
$$

Simulation and results: With the use of mandatory band group 1 with preamble 1 and TFC 1, simulation is performed under the target channel models CM1 and CM2; CM1 reflects the line of sight (LOS) case, whereas CM2 signifies the non-LOS environment. For picking out different time-related parameters, for example, sampling frequency, FFT size (i.e. $N$ ), length of zero padded suffix/number of guard samples (ie, $L$ ), we exactly follow the standard ECMA-368. Note that $L$ is composed of $L_{\text {suf }}=32$ and $L_{\text {pre }}=5$. Simulation results are established from the average of 1000 realisations.

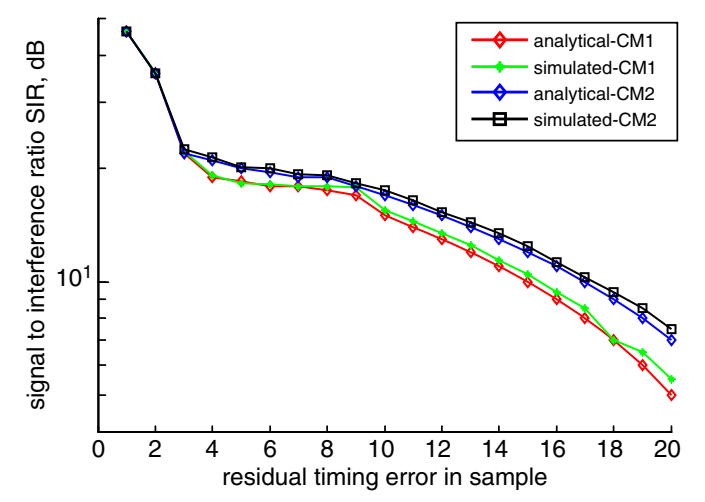

Fig. 1 Effect of residual timing offset

Based on the above simulation scenario, we achieve the following results. Fig. 1 presents the effect of residual timing offset in terms of SIR. This Figure indicates that SIR performance is extremely sensitive to residual timing error. This is a notable difference between the performances of CP-based and ZP-based systems. There is neither interference nor extra noise for CP-based systems, but there is always possible interference for ZP-based systems. We see that SIR is drastically reduced to below $15 \mathrm{~dB}$ as soon as there is a timing error of 10 samples for the CM1 environment. In the case of CM2, the SIR falls below $15 \mathrm{~dB}$ at the time of being a timing error of eight samples. Without loss of generality, it can be concluded that a timing error of more than eight samples must not be left uncompensated. A slightly better SIR performance occurrence in CM2 case is viewed. This is because the mean channel energy of CM2 realisation is little higher than that of the CM1 case. However, when the timing error is very small, these two channel models provide almost the same SIR performance because SIR is too high to dominate the difference in channel energy. Both simulated and analytical results are well matched across the entire range of residual timing error for both CM1 and CM2 environments.

Conclusion: In this Letter, we have analytically derived an expression of SIR in terms of fine timing offset. We have shown that, unlike a CP-based system, residual timing error in a ZP-based system causes possible interference or extra noise which eventually can result in higher bit error rate. This study is worthwhile for designing a practical high-speed UWB receiver.

Acknowledgment: This work was supported by the National Research Foundation of Korea (NRF) grant, funded by the Korean government (MSIP) no. 2010-0018116.

(C) The Institution of Engineering and Technology 2015

9 November 2014

doi: $10.1049 / \mathrm{el} .2014 .3967$

One or more of the Figures in this Letter are available in colour online.

S.M.R. Islam and K.S. Kwak (Wireless Communications Research Center, Inha University, 253, Yonghyun-dong, Nam-Gu, Incheon 402751, Republic of Korea)

凶-mail: riaz@inha.ac.kr

S. Ullah (CISTER, Polytechnic Institute of Porto, Porto, Portugal)

J. Lloret (Polytechnic University of Valencia, Valencia, Spain)

N. Ullah (Postgraduate Jahanzeb College Swat, Pakistan)

\section{References}

1 Li, Y., Minn, H., and Rajatheva, R.M.A.P.: 'Synchronization, channel estimation and equalization in MB-OFDM systems', IEEE Trans. Wirel. Commun., 2008, 7, (11), pp. 4341-4352

2 Yak, C.W., Ling, L.Z., Chattong, S., and Tjhung, T.T.: 'Timing synchronization and frequency offset estimation for ultra-wideband (UWB) multiband OFDM systems'. IEEE PIMRC, Berlin, Germany, September 2005, pp. 471-475

3 Yoon, S.H., and Chong, J.W.: 'Packet detection and symbol timing synchronization algorithm for multiband OFDM UWB', IEICE Trans. Commun., 2006, E89-B, (4), pp. 1433-1435

4 Berger, C., Zhou, S., Tian, Z., and Willett, P.: 'Precise timing for multiband OFDM in a UWB system'. IEEE ICUWB, Waltham, MA, September 2006, pp. 269-274

5 Schmidl, T.M., and Cox, D.c.: 'Robust frequency and timing synchronization for OFDM', IEEE Trans. Wirel. Commun., 1997, 45, (12), pp. $1613-1621$

6 Larson, E.G., Liu, G., Li, J., and Giannakis, G.B.: 'Joint symbol timing and channel estimation for OFDM based WLANs', IEEE Commun. Lett., 2001, 5, (8), pp. 740-742

7 Minn, H., Bhargava, V., and Lataief, K.: 'A robust timing and frequency synchronization for OFDM systems', IEEE Trans. Wirel. Commun., 2003, 2, (4), pp. 822-839

8 Mattera, D., and Tanda, M.: 'Blind symbol timing and CFO estimation for OFDM/OQAM systems', IEEE Trans. Wirel. Commun., 2013, 12, (1), pp. 268-277

9 Morelli, M., and Moretti, M.: 'Joint maximum likelihood estimation of CFO, noise power, and SNR in OFDM systems', IEEE Wirel. Commun. Lett., 2013, 2, (1), pp. 42-45

10 Cho, Y.H., and Park, D.J.: 'Timing estimation based on statistical change of symmetric correlator for OFDM systems', IEEE Commun. Lett., 2013, 17, (2), pp. 740-742

11 Mastofi, Y., and Cox, D.C.: 'Mathematical analysis of impact of timing synchronization on the performance of an OFDM system', IEEE Trans. Commun., 2006, 54, (2), pp. 226-230

12 Zhou, Y., Karsilayan, A.I., and Serpedin, E.: 'Sensitivity of multiband ZP-OFDM ultra-wide-band and receivers to synchronization errors', IEEE Trans. Signal Process., 2007, 55, (2), pp. 729-734 